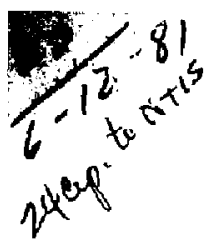

$M \cdot \varepsilon \cdot Q$

$$
\text { R.4982 }
$$

UCID- 19055

\title{
MATHEMATICAL MODELS FOR THE EPIC CODE
}

\author{
H.L. Buchanan
}

June 3,1981

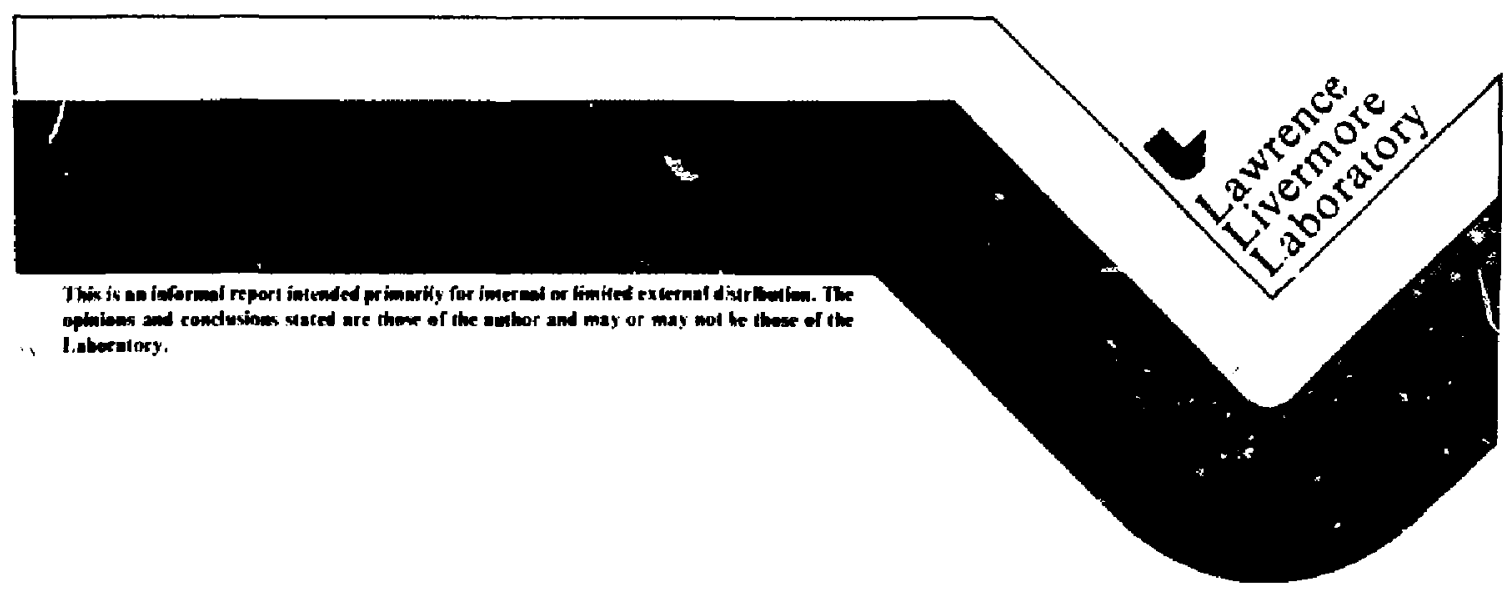

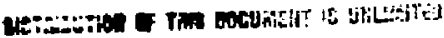




\title{
MATHEMATICAL MODELS FOR THE EPIC CODE:
}

\author{
H.L. Buchanan
}

Abstract

EPIC is a riuid/envelope type computer code designed to study the energetics and dynamics of a high energy, high current electron heam passing through a gas. The code is essentially two dimensiona $(x, r, t)$ and assumes an axis ymmetric beam whose r.m.s. radius is governed by an envolope nudel. Electromagnetic fjelds, background gas chemistry, and gas hydrodynamics (density cliannel evolucion) are all calculated self-consistent ly as functions of $t, x$, and $t$. The code is a collection of five major subroutines, each of which is described in some detail in this report.

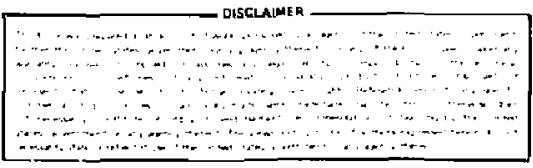

*The Lawrence Livermore National Laboratory is operated by the University of California for the Department of Energy under Contract No. W-7405-Eng-48.

This work is performed by LLNL For the Department of Defence under DARPA (DOD) ARPA Order 3718 , Amendement $\$ 33$ and 34 , monitored by NSWC under Contract No. N60921-8I-LT-WG009, Amendment No, I and N60921-81-LT-W0026. 
The computer code EPIC (Electron hlse Interactions with a Channel) was designed as a tool to be used to study the axisymetric energetic interaction of a series of arbitrarily spaced electron pulses (referred to here as a bolt) with a dynamic background gas. A desire to treat a wide range of pulse separation times forces the inclusion of a large number of physical phenomena, not all of which will apply to any single situation. For example, a high current, high energy electron pulse will ionize the background gas at a very rapid rate. "Tiss ionizdion bili cerainily constrain the evolution of the electromagnetic self-fields of the pulse and so must be followed on a sub-rianosecond time scale. On the other hand, the decay of this ionization and the attendant conductivity is essentially a chemical kinetic process and takes place over a muct longer time scale, perhaps over microseconds. Longer still are the time scales over thich the background gas can respond hydrodynamically, tens of microseconds, and yet this hydrodynamical density development significantly affects the themistry of the gas wich in turn couples into the beam self-field. In the past a particular time scale regime has been selected for examination and processes taking $\mathrm{place}$ much faster are regarded as instantaneous while slower processes are regarded as static. EPIC represents ant at temp at a more self consistent treatment of the whole beam-gas interaction problem.

The formulation is axis ymetric and as such it can shed no light on trangvirse phenomena (tracking, hose instability, etc.). Rather it is poped that the energetics of successive pulses in an evolving gas channel may be examined in some detail. In particula, we would hope to explore macrojcopic phenomena such as beam pulse erosion, pulse to pulse head expansion, joule heating of the background gas, intra-pulse conductivity build up and decay, 
and overall energy loss scaling. This list of objectives, al though partial, is already ambitious and calls for a slight departure from tr.aditional computational technique. The code EPIC shouId not be regarded as a user oriented production code, but rather a loosely coupled set of mutually compatible subroutines which can be combined like building blacks to apfly to the particjar situation at hand. There are, in fact, five major subroutine packages, each of wich advance one or more related quantities a single step in time at each call. The design of the main routine depends on the situation under examination. While this procedure will seldom optimize the computation of any one problem it has the advantage of great flexibility with only a smaIl investment of labor.

This report describes each of the five sections in terms of the overalt model used with the caveat that even the routines themse 1 ves may change in response to unique circumstances. The sections are described loosely in the order of increasing time scale beginning with the electromagnetic field solver, ASOLVE, which must obviously treat quantities changing at light speed; then slcsol, which treats conductivity, electron density, and electron temperature; RSOLVE, which calculates beam radius, energy, and emittance as a function of distance, $z$; TGSOLVE, which uses the thermodynamies of the background to calculate the gas temperture; atid finally FuUt, which follows the hydrodynamics of the background gas to calculate a gas density as a frnction of radius. The functional relationship of these quantities is depicted in fig. 1 .

Actual numerical detaii is omitted except were a particular technique may be significant in the interpretation of a result. In particular, we thay describe the overall code as two dimensional and Eulerian (fixed radial 
grid) but with two distinct radial grids in use. In routines Asolve, SIGGOLVE, and TGSOLVE an expanded center approach is used in which the calculational grid is uniform in the variable $v=1 n(1+r)$. This allow fine gridding near the beam where quantities are required with accuracy and fewer grid points near the maximum radius there the electrical boundary condition is appiied. The FLUID routine uses uniform gridding allowing for the fact that hydrodynamic development far from the beam may be important at the beam at soue later time, hence no region of space deserves particular enphasis. The roufine $\mathrm{RSOLVE}$ deals in beam averaged quantities and requires no radial reselution.

ASOIVE

As in many reient beam propagation codes, the electromagnetic field in EPIf is described by a single variable $A=A_{z}+\phi$ where $A_{z}$ and $\phi$ are components of the traditional vector and scalar potentials. As described in reference ( 1 ), this quantity gives, to an excellent approximation in relativistic beam geometry, the electric fields, $E_{z}$ and $E_{r}$, (at fixed spaizizis asordinatz, z) zs

$$
\begin{aligned}
& E_{z}=-\frac{\partial A}{\partial x} ; \quad E_{5}=-\frac{c}{4 \pi \sigma} \frac{\partial^{2} A}{\partial r \partial x} \\
& (x=c t-z)
\end{aligned}
$$

and the transverse forcs (due to electric and magnetic fields) as 


$$
F_{\mathbf{r}}=q \quad \frac{\partial A}{\partial \mathbf{r}}
$$

This new potential evolves in $x$ (a doppler shifted time) according to

$$
\frac{1}{r} \frac{\partial}{\partial r} \times\left(1+\frac{c}{4 \pi \sigma} \frac{\partial}{\partial x}\right) \underset{\frac{\partial}{\partial r}}{ } A=\frac{4 \pi}{c}\left(\sigma \frac{\partial A}{\partial x}-J_{b}\right)
$$

where we have assumed that Ohm's law is valid for the plasma return current $J_{P}=\sigma E_{z}$ and that an omitted term in $\frac{\partial^{2} A}{\partial x^{2}}$, which is part of the displacement current, is negligible. This equation is third order (two orders in $x$ and one in $x$ ) and non-linear since 0 will depend in a complicated way on the Fields. Further, we ll-posedness requires on ly the single baundary zond it i on

$$
A(r=b)=0
$$

That this is true can be seen by transforming the problem to $x, y$ space and noting that the above boundary condition gives $A$ at both $x$ and $y$ boundaries. Differencing of the field equation in a time $(x)$ centered implicit way yields a simple tri-diagonal inversion matrix whirh is easily solved by conventional methods. Stability of the numerical scheme is assured regardless of step size but step size is constrained by accuracy considerations. The expanded center feature is accomplished by changing variables to

$$
u=\ln (1+r)+r=\left(e^{u}-1\right) ; \quad \frac{\partial}{\partial x} \rightarrow e^{-u} \frac{\partial}{\partial u}
$$


This introduces variable coefficients to each derivative term but these coefficients need to be calculated only once since the u-grid is fixed. In final form

$$
\frac{\partial}{\partial u} s\left(\frac{\partial}{\partial u} A+\frac{c}{4 \pi \partial} \frac{\partial}{\partial u} \frac{\partial A}{\partial x}\right)=-\frac{4 \pi}{c} T\left(J_{b}-\partial \frac{\partial A}{\partial x}\right)
$$

where $S=1-e^{-u}$

and $\quad T=e^{u}\left(e^{u_{-1}}\right)$

\section{SIGSOLVE}

The subroutine SIGSOLVE advances the electrical conductivity, $\sigma(r)$, one time step. The conductivity is a function of both the new electron temperature and the new electron density so these quantities must be advanced as wel1. To determine electron temperature we take the energy equation to be

$$
\begin{aligned}
\frac{3}{2} n_{e} k \frac{d T_{e}}{d t}= & O E_{z}^{2}+J_{b} n_{g} k(7 e v)-n_{e} v_{i}(15 e v) \\
& -\frac{3}{2} n_{e} k\left(T_{e}-T_{g}\right) v_{u} n_{g}
\end{aligned}
$$

The first term on the right hand side is simply the joule heating of the electrons by the induced electric field. This energy is simultaneously added to the gas under the assumption that the electrons cool against the gas in a time short compared to a hydro-time. The second term models the fact that knock-on electrons resclting from collisions of beam particles with gas 
molecules are genetated with an average energy of $7 \mathrm{ew}$. The constant $k$, gives the number of electrons generated in gas of density $\mathrm{n}_{\mathrm{g}}$ according to the measured stopping power $\mathrm{dE} / \mathrm{dx}=2.52 \mathrm{Mev} / \mathrm{g} / \mathrm{cm}^{2}$. It is calculated to be

$$
K=\frac{d E / d x}{\pi E e}=1.554 \times 10^{14}\left[\mathrm{~cm}^{2} / \mathrm{g} \text { esu }\right]
$$

where we have assumed the $33.75 \mathrm{eV}$ is required for each ion pair. The third term accounts for the fact that electrons created by avalanche break-down acquire an average of $15 \mathrm{ev}$ of potential energy at the expense of the available kinetic energy. The last term models the exchange of energy from hot electrons to gas molecules by collisions. The energy exchange frequency, $v_{u}$, is taken to be a simple straight line approximation to experimental data ${ }^{4}$ shown in fig. 3. of the form

$$
u_{u}=\left\{\begin{array}{lll}
1.731 \times 10^{-11} & \sec ^{-1} \mathrm{~cm}^{3} & T_{e}<1200^{\circ} \mathrm{K} \\
1.203 \times 10^{-17} \mathrm{~T}_{\mathrm{e}}^{2}\left[0_{\mathrm{K}}\right] \sec ^{-1} \mathrm{~cm}^{3} & \mathrm{~T}_{\mathrm{e}}>1200^{\circ} \mathrm{K}
\end{array}\right.
$$

Electron density is a balance between production by collisional and avalanche ionization and depletion by ionic tecubination. We model the gas chemistry by following only two apecies. For air at moderately high temperatures we describe the chemistry in terms of two reactions

$$
\begin{aligned}
& \mathrm{e}^{-}+\mathrm{N}_{2}^{+} \rightarrow \mathrm{N}+\mathrm{N} \quad \text { (dissociative recombination) } \\
& \mathrm{e}^{-}+\mathrm{O}_{2}+\mathrm{O}_{2} \leftrightarrows \mathrm{O}_{2}+\mathrm{O}_{2}^{-} \quad \text { (attachment) }
\end{aligned}
$$


The rate equations are then written

$$
\begin{aligned}
& \frac{d n_{e}}{d t}=s_{e}-\alpha n_{e}\left(n_{e}+n_{0_{2}^{-}}-\beta n_{e}+\beta^{\prime} n_{o_{2}^{-}}\right. \\
& \frac{d n_{0_{2}^{-}}^{-}}{d t}=s_{0_{2}^{-}}-\alpha n_{o_{2}^{-}}\left(n_{e}+n_{o_{2}^{-}}\right)+\beta n_{e}-\beta \cdot n_{o_{2}^{-}}
\end{aligned}
$$

where the reaction rates are taken to be 4

$$
\begin{aligned}
& x=2 \times 10^{-7}\left(\frac{300}{T_{e}}\right)^{1 / 2} \\
& {\left[\frac{\mathrm{cm}^{3}}{\sec }\right]} \\
& B=4.04 \times 10^{8}\left(\frac{300}{T_{e}}\right) e^{-600 / T_{e}}\left(\frac{\rho_{g}}{\rho_{0}}\right)^{2} \quad\left[\frac{1}{\sec }\right] \\
& 3^{\prime}=1.61 \times 10^{9}\left(\frac{\tau_{\mathrm{e}}}{300}\right)^{1 / 2} \mathrm{e}^{-5590 / \mathrm{T}} \mathrm{e}\left(\frac{\rho_{\mathrm{g}}}{\rho_{\mathrm{o}}}\right) \quad\left[\frac{\mathrm{l}}{\mathrm{sec}}\right]
\end{aligned}
$$

$\mathrm{S}_{\mathrm{e}}$ and $\mathrm{S}_{\mathrm{O}_{2}}$ - represent source terms due to ionization by the beam, hydrodynamic convection of the gas, etc. We have then three coupled, non-1ineat equations to solve for $\mathrm{T}_{e}, \mathrm{n}_{e}, \mathrm{n}_{\mathrm{O}_{2}}{ }^{-}$. Numerically, the problem is even more complicated since the coupled set is stiff, that is it contains several widely differing time scales which must be treated simultaneously. Rather than address the numerical difficulties, we obtain approximate analytic solutions of these three equations in EPIC. 
We begin with eq. (B.1) and lump all source terms together to be held constant throughout one time step. For temperatures greater than $1200^{\circ} \mathrm{K}$ we may write

$$
\frac{d T_{e}}{d t}=-a\left(T_{e}-T_{g}\right) T_{e}^{2}+b
$$

where $b=\frac{\sigma E_{z}^{2}+J n_{g} k(7 e v)-n_{e} \nu_{i}(15 e v)}{\frac{3}{2} n_{e} k}$

$$
a=1.203 \times 10^{-17}
$$

if we now let $T=\frac{T_{e}}{T_{g}}, x=\frac{b t}{T_{g}}$, and $\xi=\frac{a T_{g}^{3}}{b}$ our equation becomes

$$
\frac{d T}{d x}=-\xi T^{2}(T-1)+1
$$

we set immediately that the steady state remperature $T^{*}$ satisfies

$$
T^{2}\left(T^{*}-1\right)=1
$$

if we now redefine $T \rightarrow \frac{T}{T^{*}}$ we ubtain

$$
\frac{d T}{d x}=-\frac{1}{\left(T^{*}-1\right)} T^{2}\left(I-\frac{1}{T^{*}}\right)+\frac{1}{T^{\star}}
$$


Depending on the coefficients $a$ and $b, T_{e}$ is either incteasing or decreasing towards $T^{*}$. For a decaying solution let $T=1+y$ to obtain

$$
\frac{d y}{d x}=-y \quad\left[\frac{3 T^{*}-2}{T^{*}\left(T^{*}-1\right)}\right]-y^{2}\left[\frac{3 T^{*}-1}{T^{*}\left(T^{*}-1\right)}\right]-y^{3}\left[\frac{1}{T^{*}-1}\right]
$$

for a growing solution we let $T=1-y$ and obtain the same equation except for the sign of the middle term. For decaying solutions $0<y<\infty$ and we make the approximation that when $y$ is large its slope is goverened by the last term and when $y$ is small its slope is determined by the first. The middle term is dropped and we obtain

$$
\frac{d y}{d x}=-\frac{y}{(T *-1)} \quad\left[\frac{3 T^{*}-2}{T^{*}}+y^{2}\right]
$$

This may be integrated over one $\Delta \mathrm{x}$

$$
y_{n+1}^{2}=\frac{c \psi \alpha^{2}}{(1-C \psi)} \quad \psi=\exp \left[-\frac{2 \alpha^{2} \Delta x}{\left(T^{*}-1\right)}\right]
$$

where $\quad \alpha^{2}=\frac{\left(3 T^{*}-2\right)}{T^{*}} \quad$ and $\quad c=\frac{y_{n}^{2}}{\alpha^{2}+y_{n}^{2}}$

A slightly better approximation to the original equation is obtained by changing the factor of 2 in $\psi$ to 3 . This partially compensates for the missing middle cerm.

For growing solutions $y$ is bounded as $0<y<1$ and a different procedure is used. Returning to the original equation (changing th s sign of the middle 
term) we replace $y^{3} \rightarrow y^{2} y_{n}$ where $y_{n}$ is, again, $y$ at the beginning of the $\Delta x$ step. We then obtain an equation that we can integrate and the solution is

$$
\frac{y}{n+1}_{a}=c \exp \left[\frac{\left(3 T^{*}-2\right) \Delta x}{\left(T^{*}-1\right) T^{*}}\right]+1
$$

where

$$
c=1+\frac{\alpha}{y_{n}} \quad \text { and } \quad \alpha=\frac{3 r^{*}-2}{\left(3-y_{n}\right)^{2}-1}
$$

1. ance through our definition of $T=I \pm y$ we can calculate $T_{e}^{n^{+l}}$ from $T_{e}^{n}$.

To solve the rate equations we first set the source terms to zero. In ou: scheme, we will apply our sources to $\mathrm{n}_{e}$ and $\mathrm{n}_{\mathrm{O}_{2}}{ }^{-}$, separate $\mathrm{l}_{\mathrm{y}}$, half at the beginning and half at the end of each time step thus simplitying our probiem a bit. The homogeneous equations are still coupled and still non-linear but an exact solution can be found as

$$
\begin{aligned}
& n_{e}^{n+1}=\Delta\left[\frac{\beta^{\prime}\left(n_{e}^{n}+n_{0}^{n-}\right)}{\left(\beta+\beta^{\prime}\right)}(1-\phi)+n_{e}^{n} \phi\right] \\
& n_{0}^{n-1}=\Delta\left(n_{e}^{n}+n_{0_{2}^{-}}^{n}\right)-n_{e}^{n+1}
\end{aligned}
$$




$$
\Delta=\frac{1}{1+\alpha \quad\left(n_{e}^{n}+n_{o_{2}}^{n}\right)} \Delta t \quad \phi=\exp \left[-\left(\beta+\beta^{\prime}\right) \Delta t\right]
$$

Note that in order to solve the equation for $T_{e}$ we had to assume ${ }_{e}$ was constant :hroughout the step and to solve for $\mathrm{n}_{\mathrm{e}}$ and $\mathrm{n}_{\mathrm{O}_{2}}$ - we had to assume $T_{e}$ was constant thraughout the step. Actually, in regions of no beam and no electric fieid $T_{e}=T_{\text {gas }}$ and $T_{e}$ changes very litele with time or $n_{e}$ so there is no difficulty. In regions of high field or large current the final values of $n_{e}$ and $T_{e}$ at $t+\Delta t$ is obtained by iterating on the analytic solutions.

Knowing $n_{e}(t+\Lambda t)$ and $T_{p}(t+\Delta t)$ we may at last calculate the conductivity according to

$$
u(t+\Lambda t)=\frac{2.54 \times 10^{8} \mathrm{n}_{\mathrm{e}}(t+\Delta t)}{v_{\mathrm{m}}+\frac{\left.37.5 \mathrm{n}_{\mathrm{e}}^{(t+} \Delta t\right)}{\mathrm{T}_{\mathrm{e}}^{3 / 2}}}
$$

where the collision frequency is given by another straight line fit to experimental data ${ }^{5}$ shown in fig. 4

$$
v_{m}= \begin{cases}2.318 \times 10^{8}\left(\frac{\Omega}{\rho_{0}}\right) \mathrm{T}_{\mathrm{e}} & \mathrm{T}_{\mathrm{e}}<19726^{\circ} \mathrm{K} \\ 4.573 \times 10^{12}\left(\frac{\rho}{\rho_{\mathrm{o}}}\right) & \mathrm{T}_{\mathrm{e}}>19726^{\circ} \mathrm{K}\end{cases}
$$

We have thus far neglected any mention of any dependence on $r$. In fact, dependence on $r$ atises predominately through the heating profiles of $\sigma E_{z}^{2}$ 
and $k \mathrm{ng}_{\mathrm{g}} \mathrm{J}_{\mathrm{b}}$. In addition we thust account for the fact that in order to maintzin charge neutrality, plasma electrons must convect radially outward with the expanding gas. This will take place on a time scale slow compared to electron heating and is therefore ignored in the temperature equation. Ths time scale for the gas chemistry is much longer, however and the source terms berome

$$
\begin{aligned}
& s_{e}=r_{g} n_{g} J_{b}+n_{e} v_{i}\left(\frac{E_{z}}{\bar{P}}\right)+\frac{n_{e}}{n_{g}} \frac{\partial n_{g}}{\partial t} \\
& s_{0_{2}^{-}}=\frac{n_{e}}{n_{g}} \frac{\partial n_{g}}{\partial t}
\end{aligned}
$$

As previously described, the first electron source term represents knock-ons (beam gas collisions). The second term describes the generation of electrons due to the avalanche ionization. This pracess is modeled as a rate $v_{i}$ which seales with $E_{z} / P$ and is taken to be ${ }^{6}$

$$
v_{i}=\frac{A S^{3}}{1+B S+C S+D S^{3}} \quad P
$$

where $S=\frac{E_{z}^{2}}{P^{?}} \quad\left(E_{z}\right.$ in statvolts and $P$ in atmospheres $)$

$$
\begin{aligned}
& A=1.423 \times 10^{-4} \\
& B=9.179 \times 10^{-6} \\
& C=2.656 \times 10^{-10} \\
& D=2.820 \times 10^{-17}
\end{aligned}
$$


The terms proportional to $\frac{\partial n}{\partial x}$, a quantity returned from the hydrodynamic alogrithm, models the depletion of electrons and ions that are carried away with expanding gas.

\section{TGSOLVE}

The subroutine TGSOLVE advances the gas temperature, $\mathrm{T}_{g}(r)$, one time step by integrating an energy equation of the form

$$
\begin{aligned}
n T_{g} \frac{d S}{d t} & =\operatorname{lr} T_{g}\left(\frac{\partial S}{\partial t}+v \frac{\partial S}{\partial r}\right) \\
& =0 E_{z}^{7}+r n_{B} J_{b}(33 e v)-\frac{40 S_{B}{ }^{4}{ }^{4}}{\ell}+\frac{1}{r} \frac{\partial}{\partial r} r k_{t h} \frac{\partial}{\partial r} T_{g}
\end{aligned}
$$

Here $\mathrm{T}_{\mathrm{g}}$ is tîe gas temperature and $\mathrm{s}$ is the entropy per gram of the gas. The first term on the right hand side results from the jaulr heating of che gas by the induced plasma current, the second term describes the direct deposition of energy in the gas by beam particles, and the final term represents themal conduction of energy in the radial direction. The direct deposition coefficient $k$ has been described previausly and the thermal conduction coefficient, $k_{t h}$, is obtained from tables ${ }^{4}$ as a function of gas temperature and pressure. It is shown in fig. 6 . The third term models energy loss due to radiation in the approximation that the gas channes is at all points optically thin. A Planck mean length is found from a look-up table as a function of gar temperature and density and is, like $k_{t h}$, implicity a function of cadius. The planck mean length is graphed in fig. 5 . 
In order to obtain an equation for the gas temperature, $T_{g}$, we need an expression for the entropy. Here the code offers two options; the first. the lorg time option, assumes that the gas is always at chemical qquilibrium, i.e. that at any particular remperature and density the gas instantly takes on its equilibrium fractional ionization. This is achieved by analytically fitting a table of entropy values measured at equilibtiun to obtain the dimensionless form

$$
\frac{S}{R_{a}}=0.289\left(\frac{T_{g}}{o_{K}}\right)^{0.5}\left(\frac{0}{\sigma_{0}}\right)^{-0.12}
$$

$R_{a}$ is the universal gas constant times the molecular weight, When this expression is substituted into the gas temperature equation a second order PDE is obtained in terms of known quancicies.

The second thermodynamic opcion involves the assumption that the gas had had insufficient time to change its composicion (in particular, particle number) and is calied the short time oprion. Entropy is now given by the Sacur-Tetrode expression for diatomic gases and is written

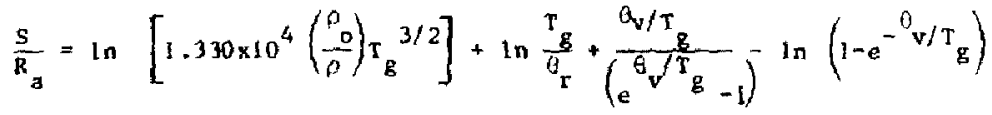

The first term corresponds to the translational contribucian, the second is dise to rotations where $\theta_{x}$ is the rotational temperature (for $\mathrm{N}_{2}, \theta_{r}=$ $2.88^{\circ} \mathrm{K}$ ), and the last two terus describe the vibrational contribution (for $N_{2}, \theta_{v}=3374^{\circ} \mathrm{K}$ ). Using this expression ve find that 


$$
\frac{\partial}{\partial t}\left(\frac{S}{R_{a}}\right)=\frac{C_{v}}{R_{a}}\left[\frac{1}{T} \frac{\partial T}{\partial t}-\left(\frac{\rho_{0}}{\rho}\right) \frac{\partial}{\partial t}\left(\frac{\rho}{\rho_{o}}\right)\right]
$$

and a similar expression for $\frac{\partial}{\partial r}\left(\frac{S}{R_{a}}\right)$

where the heat capacity $\left.\frac{c_{v}}{R_{a}}=\frac{5}{2}+\left(\frac{\theta^{v}}{T}\right)^{2} \frac{e^{\theta_{v} / T_{g}}}{\left(e^{\theta_{v} / \tau_{z}} B_{-1}\right.}\right)^{2}$

is the normal value for ideal diatomic nolecules, a function of t-mperature only. If instead we had used the long time option the result would have been

$$
\frac{\partial}{\partial t}\left(\frac{S}{R_{a}}\right)=\frac{C_{v}}{R_{a}} \frac{1}{T} \frac{\partial T}{\partial t}+0.240 \frac{C_{v}}{R_{a}}\left(\frac{\rho_{g}}{\rho}\right) \frac{\partial}{\partial t}\left(\frac{\rho}{\rho_{o}}\right)
$$

whe $\frac{C_{v}}{R_{a}}=.1445\left(\frac{\rho}{\rho_{0}}\right)^{-0.12}\left(\frac{T}{o_{K}}\right)^{0.5}$ which is now a weak function of

density as well as temperature. Comparison of the two values of $c_{v}$ at room

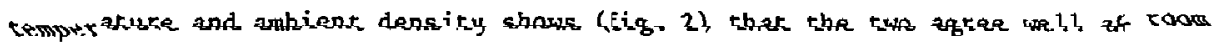
temperature and ambient density but differ by an urder of magnitude ar high temperatures. A final change of variable from $r$ to $u=\ln (d+r)$ yields the gas temperature equation in the short time optian as

$$
\begin{aligned}
& c_{v} n \frac{d T}{d t}-R_{a} T \frac{d O}{d t}=\sigma E_{z}^{2}+k n_{b} J_{b}-\frac{4 \sigma S_{S B_{E}^{T}}^{4}}{\ell} \\
& +\frac{1}{e^{u}\left(e^{u}-1\right)} \frac{\partial}{\partial u}\left(1-e^{-u}\right) k_{t h} \frac{\partial}{\partial u} T_{g}
\end{aligned}
$$


where $\frac{d}{d t} \rightarrow \frac{\partial}{\partial t}+v\left(1-e^{-u}\right) \frac{\partial}{\partial u}$. The numerical differencing of this equation is accomplished according to the explicit method of Dufort-Frankel to insure stability independent of step size.

\section{FLUID}

The subroutine FLUID advances the gas density $\rho(r)$ and the radial gas velocity $v(r)$ one time step according to a conventional fluid model consisting $G E$ in equation of motion (Eulerian)

$$
o\left(\frac{\partial v}{\partial t}+v \frac{\partial v}{\partial r}\right)=-\frac{\partial}{\partial r} P
$$

and an equation of continuity

$$
\frac{\partial p}{\partial t}+\frac{1}{r} \frac{\partial}{\partial r}(r \rho v)=0
$$

where $\beta$ is the gas mass density, $v$ the radial velocity, and $p$ the radial pressure. In order to close the set ve require an equation of state which, of course, must be consistent with the gas thermodynamic assumptions outlined during the discussion of IGSOLVE. For the two options we have the pressure (in dynes $/ \mathrm{cm}^{2}$ ) given as

$$
P= \begin{cases}2.618 \times 10^{6} \mathrm{~T}_{\mathrm{g}} p & \text { short time } \\ 3.848 \times 10^{4} \mathrm{~T}_{\mathrm{g}}^{1.463} \mathrm{\rho} 0.899 & \text { long time }\end{cases}
$$

where $T_{g}\left[{ }^{0} \mathrm{x}\right]$ and $\left[\mathrm{g} / \mathrm{cm}^{3}\right]$. It will be noticed that at standard temperarure and density the long time equation of state gives a pressure that is about one thiid normal. This is a result of the fact that the 
parameterization of eq. C.6 was fit to data for temperatures greater than $10^{4 a_{F}}$ and gives error at lower temperatures.

For cases of our interest, we expect the creation and propagation of shock-like structure to be endemic, hence careful numerical treatment is required. We have chosen to adopt a toro-step Lax-llendroff procedure with the incorporation of flux correction to the transport of both density and velocity. This procedure has been described in detail elsewhere by its invertors Book, Boris, and Hain ${ }^{9}$ under the name of Phoenical Lax-Wendroff and go will only briefly outlined here. Basically, the procedure is designed to propagate a quantity characterized by steep gradients along a fjxed Euleian grid, explicitly and stably, without introduction of spurious, numerically generated, structure on the quality. This is accomplished by adidg an artificial diffusion to the quantity to insure, prior to eacli time step, smooth transport and then removing the diffusion at the end of the step to recover the original character of the quantity. The key to the process

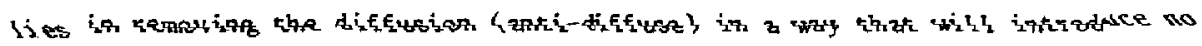
new maxima or minima in the transported quantity. As adopted in the present code, this procedure allows us to follow the highty non-linear evolution of the gas channel in the presence of intense heating by the beam and its associated fields with a modest invesment in time and space. While flux corrected transport (FCT) does tend to square of rounded edges, precise shock profiles are not of interest here.

Unlike other sections of the code, TGSOLVE works in the variable $r$ father than $u$ Bince fluid behavior at radii latge compared to the beam radius pay have a significant effect on the gas density and temperature on the axis of 
the beam, In addition, available experimental jata of gas behavior likely comes from acoustic sensors placed away from the beam center so that comparison would require accurate numerical treatment there.

\section{RSOLVE}

Propagation of beam pulses in $z$ is accomplished within the framework of an envelope model ${ }^{8}$ in which the beam profile changes self-similarly in r.m.s. radjus, $R$, according to the equation

$$
\frac{d^{2} R}{d z^{2}}+\frac{q E z}{\gamma m c^{2}} \frac{d R}{d z}=-\frac{u^{2}}{R}+\frac{E^{2}}{R^{3}}
$$

$E$ is defined to be a beam emittance, a quantity indicative in some respects of transverse beam temperature $\gamma^{2} E^{2}$ can be thought of as a transverse phase area of beam particles in a particular beam segment and can be expected to nearly constants in $z$, increasing only by scattering and non-linear effects according to

$$
\frac{d E^{2}}{d z}+\frac{2 q E_{z}^{2}}{\gamma m c^{2}} E^{2}=-2 \alpha u^{2} E \frac{\left(E^{2}-u^{2} R^{2}\right)}{\left(E^{2}+u^{2} R^{2}\right)}+\left(\frac{d E^{2}}{d z}\right)_{s c a t t}
$$

The first term on the right hand side is taken as a model of emittance increase due in the main to anharmonic betatron oscillations of the beam particles. The renult of this particular ansatz is to damp out radial beam oscillations due to this anharmonic "phase mixing" of the beam particles. The sonstant $\alpha$ (of order 0.5 ), is chosen to correspond to damping as observed in 

Finally we must model the loss of energy by beam particles themselves. We assume that all particles within a segment of the beam (rezion in the neighborhood of a fixed $x$ may be characterized by a single value of total energy $\gamma_{m^{2}}{ }^{2}$. We may then account for changes in energy by writing ior a beam of negatively charged particles

$$
\delta \frac{\partial}{\partial \tau} Y m c^{2}=-\frac{Y m c^{2}}{\lambda_{R}}-\left(\frac{\partial Y m c^{2}}{\partial z}\right) \text { scatr }-E_{z}
$$

the three terms on the right hand side rapresent losses due to Bremsirahlung, depostion of energy by the beam into the background gas, and ohmic heating of the gas. The depositional term represents a sum of the effects of many pracesses and way io approximated by the usa of a constant stopping power formula

$$
\left(\frac{\partial y m c^{2}}{\partial z}\right)_{\text {scatt }}=3.25 \times 10^{-3}\left(\frac{\rho}{\rho_{0}}\right) \frac{\mathrm{MeV}}{c m}
$$

The subroutine RSOLVE uses the above set of equations to advance $R, Y$, and $E$ one step in $z$ at a fixed $x$. It is called at the completion of each $x-s t e p$ (for which $R \neq 0$ ) and the $\mathrm{I}, \mathrm{Y}$, and $\mathrm{E}$ for that segment is immediately replaced with the advanced value. This substantially reduces the required amount of storage space. 
REFER ENCES

1. E.P. Lae, "The New Field Equations", LLNL, UCID 17286,4 Oct. 76.

2. R.R. Johnston, O.R. P1 atas, and L.M. Tannenwald, "Atomic Line Transitions and Radiation From High Temperature Air", LMSC Technical Report N-3L-TO-1, $15 \mathrm{July} 70$.

3. J.M. Yos, AVCO Report, RAD-TM-63-7 (196) (revised 1967).

4. M.H. Bortner and T. Baurer Editors-in-Chief, Defenge Nuclear Agency Reaction Rate Handbook, DNA 1948H, Genera: Electric Co., Space Division, Space Sciences Laboratory, P.0. Box 8555, Philadelphia, Penn. 19101.

5. A.G. Engelhardt, A.V. Phelps, and C.G. Risk, "Determination of Momentum Transfer and Inelastic Collision Cross Sections for Electrons in Nitrogen Using Transport Coefficients", Phys. Rev. 135, A1566 (1964).

6. P. Felsenthal and J.M. Proud, Physical Review 139 (6A) 1796 (1965).

7. Ya. B. Zel'dovich and Yu. P. Raizer, "Physics of Shock Waves and High-Temperature Hydrodynamic Phenomena Academic Press", New York and London, 1966.

8. E.P. Lee and R.K. Cooper, Particle Accelerators, Vo1. 7, PP. 83-95 (1916).

9. D.L. Book, J.P. Boris, and K. Hain, "Flux-Corrected Transport II: Generalizations of the Method", J. Conput. Phys. 18 (1975). 248-283. 


\section{Energetics}

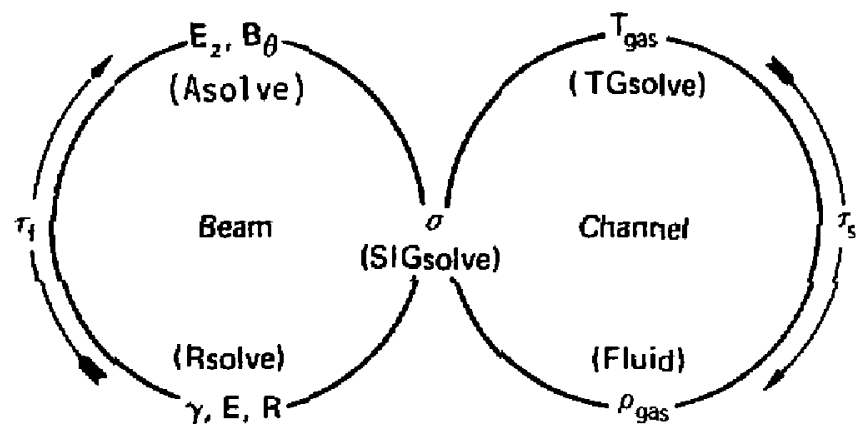

Dynamics

Fig. 1. Schematic depiction of the working relationship of the subroutines in the code EPIC. The left hand loop is advanced on a fast time scale $\tau_{E}$ while the right hand loop is advanced on a slow time scale $\tau_{s}$, 


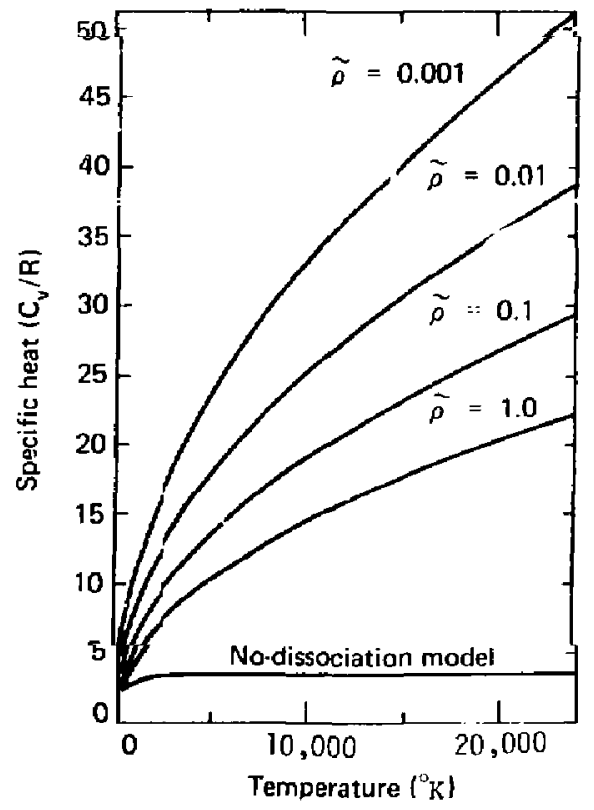

Fig. 2. Comparison of the spacific heat obtained from the two therodynamic models lescribed in the text. 


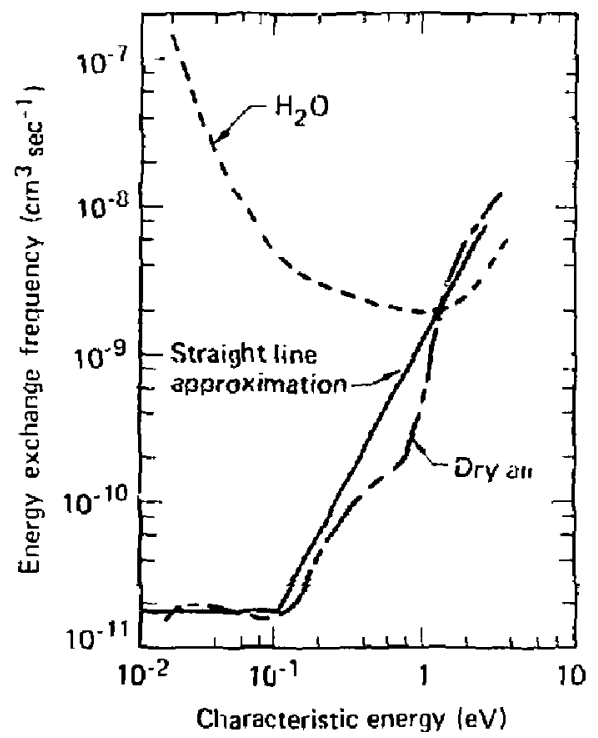

Fig. 3. The energy exchange frequency, $u$, as a function of anergy and the straight line approximation used in EPIC. 


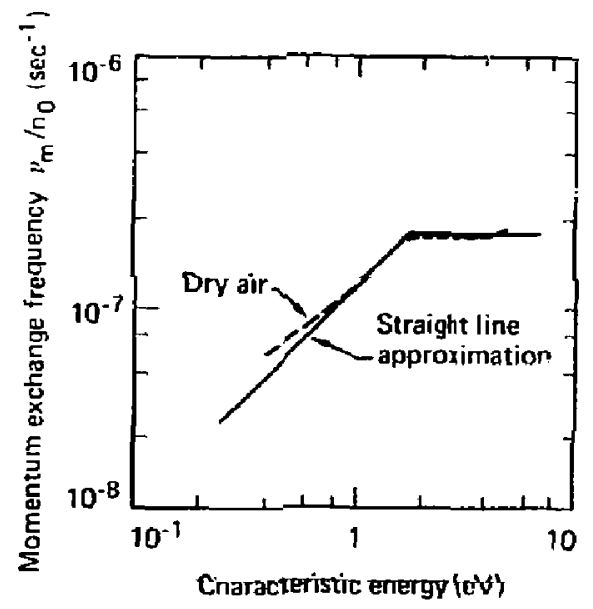

Fir. 4. The momentum exchange frequency, $\nu_{m}$, and the straight line approximation used in EPIC. 


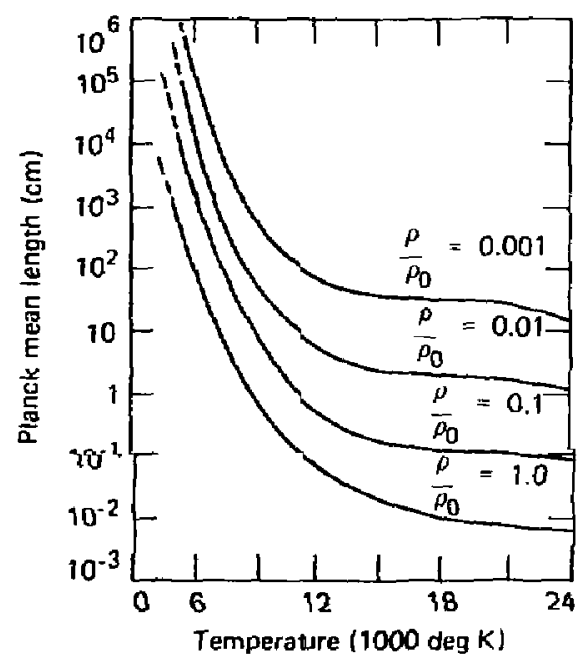

Fig. 5. The Planck mean length for air as a function of temperature and density as used in EPIC. 


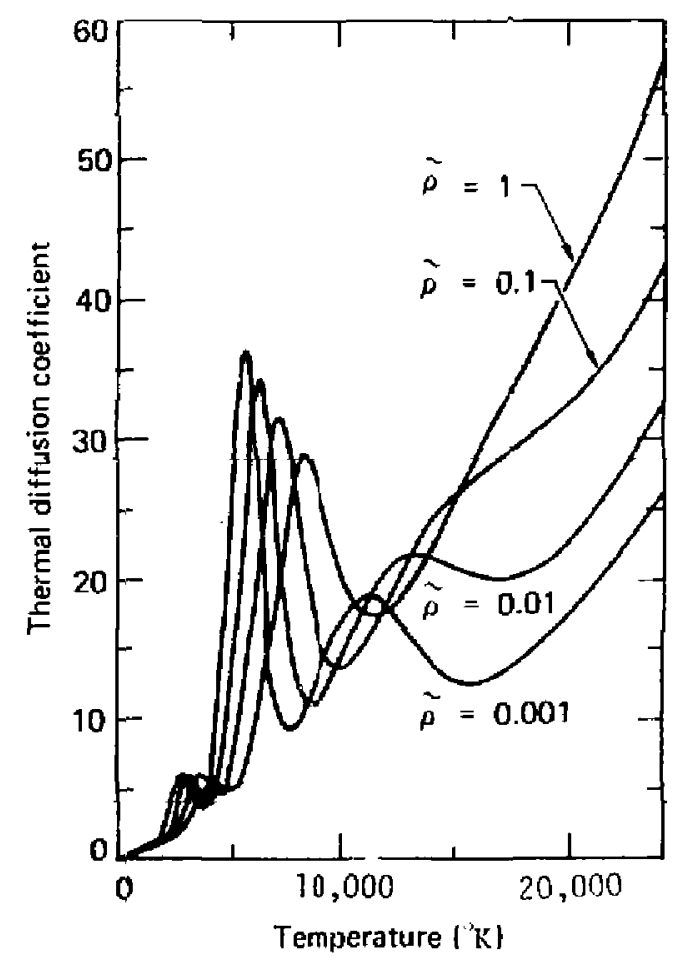

Fig. 6. The thermal diffusion coefficient $K_{t h}$ as a Eunction of temperature and densicy as used in EPIC. $K_{\text {th }}$ is given in
units of $10^{-4}$ (erg $\sec ^{-1} \sec ^{-1}$ oK-1). 